\title{
Incongruent Expression of Brain-Derived Neurotrophic Factor and Cortisol in Schizophrenia: Results from a Randomized Controlled Trial of Laughter Intervention
}

\author{
Shu-Li Cheng' ${ }^{1}$ Fu-Chi Yang ${ }^{2}$, Hsuan-Te Chu³, Chia-Kuang Tsai ${ }^{2}$, Shih-Chieh $\mathrm{Ku}^{3}$, \\ Yu-Ting Tseng ${ }^{3}$, Ta-Chuan Yeh ${ }^{4,5 *}$, and Chih-Sung Liang ${ }^{3,6 * \varpi}$ \\ ${ }^{1}$ Department of Nursing, Mackay Medical College, Taipei, Taiwan, ROC \\ 2Department of Neurology, Tri-Service General Hospital, National Defense Medical Center, Taipei, Taiwan, ROC \\ ${ }^{3}$ Department of Psychiatry, Beitou Branch, Tri-Service General Hospital, National Defense Medical Center, Taipei, Taiwan, ROC \\ ${ }^{4}$ Department of Psychiatry, Tri-Service General Hospital, National Defense Medical Center, Taipei, Taiwan, ROC \\ ${ }^{5}$ Division of Psychiatry, Penghu Branch, Tri-Service General Hospital, Penghu, Taiwan, ROC \\ ${ }^{6}$ Graduate Institute of Medical Sciences, National Defense Medical Center, Taipei, Taiwan, ROC
}

Objective Schizophrenia has been associated with dysfunction of the hypothalamic-pituitary-adrenal axis. Furthermore, alterations in neurotrophic factors might contribute to the pathogenesis of schizophrenia. We aimed to evaluate the effects of a simulated laughter intervention on the levels of cortisol and BDNF and to determine whether the effects associated with simulated laughter could be sustained after discontinuation of the intervention.

Methods In this randomized controlled study, patients with schizophrenia according to DSM-IV clinical criteria were randomly assigned to receive either 8-week-long simulated laughter intervention $(\mathrm{n}=32)$ or treatment-as-usual group (control group, $\mathrm{n}=27)$. The serum levels of BDNF and cortisol were measured at baseline, week 8, and four weeks after discontinuation (week 12) of the intervention program.

Results After an 8-week simulated laughter intervention, the laughter group had significantly higher levels of BDNF; however, four weeks after discontinuation of the intervention, the levels of BDNF significantly dropped. Interestingly, the levels of cortisol did not change significantly at week 8 , but they were significantly elevated at week 12 . The levels of BDNF and cortisol in the control group did not change significantly between week 0 and week 8 .

Conclusion These findings suggest that the simulated laughter intervention has an early effect on neurogenesis with a significant delayed effect on stress regulation in subjects with schizophrenia.

Psychiatry Investig 2020;17(12):1191-1199

Key Words Laughter therapy, Brain-derived neurotrophic factor, Cortisol, Schizophrenia, Complementary therapies.

\section{INTRODUCTION}

Laughter, a human emotional expression, is universally produced and recognized across all contexts of social interaction. Laughter is a fundamental communicative signal, promoting person-to-person bonding and even negotiating. ${ }^{1}$ While different fields of researchers have proposed several laugh types, ${ }^{2}$ laughter in essence can be classified into two types: emotionally-driven, involuntary (i.e., spontaneous) laughter and selfinduced, non-emotional, articulated (i.e., volitional or simulated) laughter. ${ }^{1}$ In recent decades, scientific evidence supports the notion that laughter is beneficial for both physical and mental health. ${ }^{3,4}$ Social laughter may further trigger endorphin release which provides a neurochemical mechanism contrib-

Received: July 12, 2020 Revised: September 12, 2020 Accepted: September 20, 2020

$\triangle$ Correspondence: Ta-Chuan Yeh, MD

Department of Psychiatry, Tri-Service General Hospital, National Defense Medical Center, No. 325, Sec. 2, Cheng-Gung Rd., Neihu District, Taipei, 114, Taiwan, ROC Tel: +886-2-87923311, Fax: +886-2-87927221, E-mail: fantine7520@ndmctsgh.edu.tw

$\triangle$ Correspondence: Chih-Sung Liang, MD

Department of Psychiatry, Beitou Branch, Tri-Service General Hospital, National Defense Medical Center, No. 60, Xinmin Road, Beitou District, Taipei City 112, Taiwan, ROC

Tel: +886-2-2895-9808, Fax: +886-2-2895-7633, E-mail: lcsyfw@gmail.com

(c) This is an Open Access article distributed under the terms of the Creative Commons Attribution Non-Commercial License (https://creativecommons.org/licenses/by-nc/4.0) which permits unrestricted non-commercial use, distribution, and reproduction in any medium, provided the original work is properly cited 
uting to the long-term maintenance of social bonds. ${ }^{5}$

Schizophrenia is a chronic mental disorder characterized by a combination of psychotic symptoms and cognitive and motivational dysfunction. ${ }^{6}$ When facing social stress, patients with schizophrenia may have impaired hypothalamic-pituitaryadrenal (HPA) axis activation. ${ }^{7}$ A blunted cortisol response in schizophrenia has been associated with more severe symptoms and worse prognosis. ${ }^{7-9}$ Several studies have used heart rate variability (HRV) to examine the imbalance of HPA axis in schizophrenia, suggesting that patients with schizophrenia had diminished HRV compared with healthy controls, such as decreased power of high frequency (HF) and low frequency (LF). ${ }^{10-14}$ This pattern of reduced complexity in heart rate fluctuations in schizophrenia suggests that these individuals experience difficulty in adapting their heart rate in response to environmental stimuli. In addition, patients with schizophrenia have decreased levels of brain-derived neurotrophic factor (BDNF), ${ }^{15,16}$ a neurotrophin that regulates neuronal survival, differentiation, plasticity, and development in the central and peripheral nervous systems. ${ }^{17}$ The reduced BDNF expression in schizophrenia may underlie the core behavioral and cognitive symptoms of this disorder. ${ }^{16,18,19}$ Importantly, previous studies suggest that the variation of peripheral BDNF levels in schizophrenia may be associated with the efficacy of pharmacological interventions. ${ }^{20}$ However, whether non-pharmacological interventions can bring about BDNF changes and produce a clinical response is still unknown.

Sufficient evidence shows the benefits of simulated laughter on physiological and psychological systems in healthy people. ${ }^{2,3}$ To date, very few studies have examined such effects in people with schizophrenia. One study assessed the effects of a humor intervention on positive and negative symptoms in schizophrenia and found not only improved in clinical symptoms of psychosis but also in rehabilitative outcomes after five weeks of humor intervention. ${ }^{21}$ Patients with schizophrenia may be the most in need of cost-efficient means to improve their social cognition and social interaction skills, ${ }^{22}$ and a laughter intervention is a non-pharmacological, easy-to-use, and inexpensive natural therapeutic modality. The aim of this study was to evaluate the effects of simulated laughter on mood regulation, self-esteem, and other physiological domains reflecting the stress response, including heart rate variability (HRV) and the peripheral levels of BDNF and cortisol. We also tested the lasting effect of a simulated laughter intervention, which has not previously been evaluated.

\section{METHODS}

\section{Study design}

We conducted a 8-week open-label randomized controlled study of Laughing Qigong Program (LQP). Subjects were randomized to either LQP group or control group. Randomization was performed according to a computer-generated schedule with a permuted-block design. The investigators did not know the block size. The person generating the randomization schedule was not involved in determining patients' eligibility, administering treatment, or the outcome assessment. The sample size estimation for intermediate effect sizes and 95\% confidence interval with continuous outcomes corresponds to approximately 60 participants. In the end, 66 participants were randomized to either the laughter group $(n=34)$ or the comparison group $(\mathrm{n}=32)$.

The experimental protocol was approved by the Institutional Review Board for the Protection of Human Subjects at the TriService General Hospital, National Defense Medical Center in Taipei, Taiwan (TSHGIRB No. BT100-08). Each subject was completely informed about the procedures. Between 2011 and 2012, participants who were undergoing rehabilitation in the chronic ward of the Beitou Branch of Tri-Service General Hospital were eligible to participate. The subjects were required to be fully capable of comprehending the study's purpose, procedures, treatments, risks and possible benefits, alternative treatments, and their right to refuse to participate. All the participants provided written informed consent and were free to withdraw their participation at any time.

\section{Study population}

The inclusion criteria were as follows: 1) age between 20 and 60 years; 2 ) meeting the diagnostic criteria for schizophrenia according to the Diagnostic and Statistical Manual of Mental Disorders, Fourth Edition, Text Revision; 3) hospitalized for more than 6 months; and 4) motivated to join the study group. Each eligible participant was randomized to either the intervention or control group.

Individuals were excluded if they had a systolic blood pressure of $>140 \mathrm{~mm} \mathrm{Hg}$ or a diastolic blood pressure of $>90 \mathrm{~mm}$ $\mathrm{Hg}$; if they had a history of a significant medical disorder, such as diabetes, cardiovascular disease, liver cirrhosis, renal disease, rheumatic arthritis, endocrinopathies or cancer; if they had a history of a neurological disorder, such as epilepsy or Parkinson's disease; if they met the criteria for substance-related disorders, including nicotine and alcohol; and if they were involved in a special physical exercise program. Betel nut chewing, cigarette smoking, and alcohol consumption were not allowed during the study.

A series of examinations were performed, including a complete physical examination, routine biochemical panel, complete blood count, urinary test, stool study, chest radiography, and electrocardiography (ECG). The height $(\mathrm{cm})$ and weight (kg) of each participant were measured using a standard bal- 
ance beam scale, and the body mass index (BMI) was calculated. Medical charts were systematically reviewed in order to confirm that all participants met the above-mentioned criteria.

\section{Laughing Qigong Program}

The LQP is a combination of qigong techniques and simulated laughter with a focus on the mind and body connection. The LQP groups were carried out for 40-50 minutes by two experienced, certified trainers. The detailed procedures for LQP have been previously reported ${ }^{23,24}$ and are briefly summarized here. The LQP is composed of four stages: 1) warm-up stage (3 min), 2) RenMai and DuMai stages (4 min), 3) transformation stage (10 $\mathrm{min}$ ), and 4) cool-down stage (5 min). All subjects were asked to complete a minimum amount of time per stage. The transformation stage consisted of two parts-the stating of emotions and simulated laughter. The stating of emotions increases awareness of emotions and current mood states and the simulated laughter transforms negative mood states. Subjects are taught to accept negative emotional states and, rather than feeling helpless or overwhelmed, actively engage in transforming them internally while in the company of others in the LQP group. The social support of other group members helps participants realize that they are not alone in feeling their negative emotions. By providing a safe context for releasing emotions, and then transforming them in a group setting, the LQP process generates a sense of empowerment.

The LQP was conducted twice a week for 8 weeks in the laughter group and participants in the control group were asked to continue their usual daily activities and did not receive any form of exercise. All the psychotropic drugs were unchanged throughout the course of the study.

\section{Physiological measures}

The laughter group was assessed at baseline $\left(\mathrm{W}_{0}\right)$, week 8 $\left(\mathrm{W}_{8}\right.$, the end of the 8-week LQP), and week $12\left(\mathrm{~W}_{12}, 1\right.$-month follow-up after the LQP). The control group was assessed at $\mathrm{W}_{0}$ and $\mathrm{W}_{8}$.

The physiological measures included the serum levels of BDNF and cortisol, weight, blood pressure, and HRV. After overnight fasting, peripheral venous blood samples were drawn by venipuncture in the morning between 07:30 and 08:30 hours. The blood samples were collected in vacutainer tubes (Venosafe, Terumo Europe N.V., Leuven, Belgium) without an anticoagulant. According to the manufacturer's instructions, samples were stored at $7^{\circ} \mathrm{C}$ for $\sim 30 \mathrm{~min}$ for deactivation of coagulation factors. The blood samples were centrifuged for 15 minutes at $1000 \times \mathrm{g}$ and $4^{\circ} \mathrm{C}$ (Rotixa 50, Hettich Zentrifugen, Mühlheim, Germany). The serum was obtained and assayed immediately or aliquoted and stored at $\leq-20^{\circ} \mathrm{C}$. The serum BDNF levels were measured using enzyme-linked-immunosorbent (ELISA) assay (BDNF R\&D Systems, Minneapolis, MN, USA) (Cat. No. DBDOO). The serum cortisol levels were measured using a commercial ELISA kit (IBL-Hamburg GmbH, Hamburg, Germany) (Cat. No. RE52061). All blood samples were run in duplicate and the average value of the replicates was taken as the sample result.

Detailed procedures of the analysis of HRV (SA-3000P HRV Analyzer, Medicore Co. Ltd., Seoul, South Korea) have been reported previously and are briefly summarized here..$^{25,26}$ The standard frequency-domain measurements were as follows: very low frequency (VLF; <0.04 Hz), LF $(0.04-0.15 \mathrm{~Hz}), \mathrm{HF}$ (0.15-0.40 Hz), total power (TP), the ratio of LF to HF (LF/ $\mathrm{HF}$ ), and LF plus HF. The spectral components of HRV were analyzed as absolute units through log transformed data (ln $\mathrm{ms}^{2}$ ). The time-domain parameters were the mean RR intervals (MRR), the mean of all normal-to-normal interbeat intervals (MNN), and the variance of the RR intervals (VAR). The time-domain parameters were measured in milliseconds (ms). All HRV analyses were performed by a trained research nurse blinded to the protocol. Individuals who showed predominantly irregular sinus rhythm or those who had sustained atrial arrhythmias, such as atrial fibrillation or $>5 \%$ ectopic complexes, were excluded from further analysis.

\section{Psychological measures}

The psychological measures included the Rosenberg SelfEsteem Scale (RSES) ${ }^{27}$ and the. Beck Depression Inventory-II (BDI-II). ${ }^{28}$

The RSES is a widely used self-report instrument for evaluating individual self-esteem in social science research. By measuring both positive and negative feelings about the self, the scale is believed to be unidimensional regarding the overall feelings of self-worth or self-acceptance. It is a short, easy to administer, Likert-scale questionnaire, with ten items answered on a four-point scale with responses ranging from strongly disagree to strongly agree. Higher scores on the RSE indicate higher self-esteem.

The BDI-II is a 21-question, multiple-choice, self-report inventory. It is one of the most widely used psychometric tests for quantifying levels of depression. Each of the 21 items is related to symptoms of depression such as hopelessness and irritability, feelings of guilt or of being punished, as well as physical symptoms such as fatigue, weight loss, and lack of interest in sex. On two items (16 and 18) there are seven options to indicate either an increase or decrease in appetite and sleep. Cut-off score guidelines for the BDI-II are given with the recommendation that thresholds be adjusted based on the characteristics of the sample and the purpose of the BDI-II. The cutoffs were: 8 , no depression; 9-13, minimal depression; 14-19, mild depression; 20-28, moderate depression; and 29-63, se- 
vere depression. All psychological measures were performed at baseline (W0) and week 8 .

\section{Statistical analysis}

Data are presented as the mean \pm standard deviation or as percentages. Group differences in continuous variables were analyzed using independent-sample t-tests. Pearson's chi-squared test was used to compare the distribution of categorical variables between groups. If the marginal distribution for the categorical variables was uneven or if there was a small value (less than five) in one of the cells, the Fisher's exact test was used. To evaluate the treatment effects of the laughter intervention compared to no intervention, we performed a two-way repeated measures analysis of variance (ANOVA) with the intervention condition as the grouping factor and pre- and post-treatment measures as the repeated measures. Treatment effects following the discontinuation of the laughter intervention were analyzed using repeated measures analysis. A Bonferroni adjustment was used to correct for multiple comparisons.

All the statistical tests were two-sided, and p-values less than 0.05 were considered significant. All statistical analyses were performed using IBM SPSS Statistics for Windows, Version 22.0 (IBM Corp., Armonk, NY, USA).

\section{RESULTS}

\section{Demographics and clinical characteristics}

Two patients in the laughter group failed to complete the LQP and five patients in the control group refused to complete the physiological and psychological measures. Data analysis consisted of 32 subjects with schizophrenia receiving LQP and 27 control subjects (Supplementary Figure 1 in the online-only Data Supplement). Table 1 shows the demographic and clinical characteristics of subjects in the laughter group and the control group. At baseline, the laughter group did not differ from the control group on the variables of age, sex ratio, education level, height, weight, or BMI. There were no significant between-group differences in levels of BDNF and cortisol. In addition, the time and frequency domain parameters of HRV in the laughter group did not differ from those in the control group.

\section{HRV, BDNF, cortisol, and other measures}

Table 2 shows the physiological and psychological measures of subjects in both groups at $\mathrm{W}_{0}$, and $\mathrm{W}_{8}$. In the laughter group, participants experienced significantly higher levels of BDNF $(\mathrm{p}<0.001)$ and LF $(\mathrm{p}=0.003)$ and significantly lower RSES ( $\mathrm{p}=$ 0.039 ) after the intervention, while there was no significant change in the control group for any of these factors (Figure 1). For the two-way ANOVAs, group effect refers to the laughter group versus control group and treatment effect for pre- versus post-treatment conditions. Two-way ANOVAs showed significant interaction effects for BDNF (Figure 2), but no significant differences for all other parameters.

Figure 3 illustrates the Bonferroni post-hoc tests for the levels of BDNF and cortisol in the laughter group. The levels of $\mathrm{BDNF}$ at $\mathrm{W}_{8}$ were significantly higher than those at $\mathrm{W}_{0}(12.1$ \pm 3.4 vs. $6.3 \pm 2, p<0.001)$, and the levels of $B D N F$ at $W_{12}$ were significantly lower than those at $\mathrm{W}_{8}(4.9 \pm 2$ vs. $12.1 \pm 3.4$, $\mathrm{p}<$ 0.001 ). The levels of cortisol at $W_{8}$ were not significantly different from those at $\mathrm{W}_{0}(13.1 \pm 3.1$ vs. $13 \pm 3.9, \mathrm{p}>0.999)$; however, the levels of cortisol at $\mathrm{W}_{12}$ were significantly higher than those at $\mathrm{W}_{8}(16 \pm 3.6$ vs. $13.1 \pm 3.1, \mathrm{p}<0.001)$ and $\mathrm{W}_{0}(13 \pm 3.9$ vs. $13 \pm 3.9, \mathrm{p}=0.022)$.

\section{DISCUSSION}

The present study showed that an 8-week simulated laughter intervention in subjects with schizophrenia could affect the levels of BDNF, RSES, and LF of HRV. Meanwhile, this intervention did not change the levels of cortisol, other parameters of HRV, or depression state. Four weeks after discontinuation of the simulated laughter intervention, the levels of BDNF decreased but the levels of cortisol increased in the intervention group. Between-group analyses showed a significant groupby-time interaction for LF, driven by increases in LF power after the simulated laughter intervention. In contrast, the control group did not show any significant changes in these psychological and physiological measures from $\mathrm{W}_{0}$ to $\mathrm{W}_{8}$. Therefore, the cortisol response stimulated by the intervention did not occur directly during the program, but rather showed elevation after four weeks without the intervention. In line with the delayed cortisol peak, the increment of LF power is more likely to represent earlier activity of autonomic nervous system than hormonal response. As a result, although patients with schizophrenia are influenced by their negative and cognitive symptoms, the LQP might still have benefits in upregulating the levels of BDNF in the short-term and sustaining its effect on the activity of the HPA axis.

Prior evidence supports that a combination training program with resistance and aerobic exercise could significantly raise the circulating BDNF levels in patients with schizophrenia. ${ }^{29} \mathrm{~A}$ meta-analysis indicated that acute and regular exercise has a significant impact on BDNF levels. ${ }^{30}$ However, BDNF serum level dropped significantly four weeks after discontinuing the intervention. Therefore, whether the LQP intervention needs a minimum duration to maintain this influence needs to be clarified.

Increased complexity in heart rate fluctuations is indicative of strong cardiac adaptability, while patients with schizophre- 
Table 1. Demographics and clinical characteristics in laughter group and control groups

\begin{tabular}{|c|c|c|c|c|}
\hline & $\begin{array}{l}\text { Laughter group } \\
\qquad \mathrm{N}=34\end{array}$ & $\begin{array}{c}\text { Control group } \\
\mathrm{N}=32\end{array}$ & $z / \chi$ & $\mathrm{p}$ value \\
\hline Age (yr) & $49.6 \pm 9.30$ & $51.7 \pm 8.00$ & 0.899 & 0.373 \\
\hline Female (N) (\%) & $14(43.8)$ & $12(44.4)$ & 0.003 & 0.957 \\
\hline \multicolumn{5}{|l|}{ Education (\%) } \\
\hline$<9$ years & $14(43.8)$ & $11(40.7)$ & 1.67 & 0.449 \\
\hline $9-12$ years & $11(34.4)$ & $13(48.1)$ & & \\
\hline$>12$ years & $7(21.9)$ & $3(11.1)$ & & \\
\hline Height (cm) & $162.4 \pm 6.8$ & $161.0 \pm 8.3$ & 0.689 & 0.493 \\
\hline Weight (kg) & $71.1 \pm 13.6$ & $69.3 \pm 15.9$ & 0.487 & 0.628 \\
\hline BMI $\left(\mathrm{kg} / \mathrm{m}^{2}\right)$ & $27.0 \pm 5.1$ & $26.7 \pm 5.6$ & 0.237 & 0.813 \\
\hline BDNF (ng/mL) & $6.3 \pm 2$ & $6.0 \pm 2.1$ & 0.576 & 0.567 \\
\hline Cortisol (ng/mL) & $13 \pm 3.9$ & $14.3 \pm 4$ & 1.268 & 0.21 \\
\hline BDI-II & $10.5 \pm 10.6$ & $10.4 \pm 9.2$ & 0.033 & 0.974 \\
\hline RSES & $22.7 \pm 1.8$ & $22.9 \pm 2.9$ & 0.214 & 0.831 \\
\hline Systolic blood pressure & $103.2 \pm 13.8$ & $104.3 \pm 16.6$ & 0.273 & 0.786 \\
\hline Diastolic blood pressure & $69.7 \pm 13.7$ & $68.1 \pm 12.4$ & 0.449 & 0.655 \\
\hline \multicolumn{5}{|l|}{ Heart rate variability } \\
\hline Heart rate & $90.7 \pm 12.3$ & $88 \pm 16.1$ & 0.727 & 0.47 \\
\hline MRR & $712.4 \pm 139.9$ & $738 \pm 99.8$ & 0.795 & 0.43 \\
\hline VLF & $4.2 \pm 1.5$ & $4.7 \pm 1.4$ & 1.155 & 0.253 \\
\hline $\mathrm{LF}$ & $2.4 \pm 2.2$ & $3.2 \pm 1.6$ & 1.854 & 0.069 \\
\hline $\mathrm{HF}$ & $2.5 \pm 1.9$ & $3.3 \pm 1.6$ & 1.707 & 0.093 \\
\hline $\mathrm{LF} / \mathrm{HF}$ & $1.8 \pm 2.6$ & $1.4 \pm 1.3$ & 0.197 & 0.844 \\
\hline $\mathrm{TP}$ & $4.7 \pm 1.6$ & $5.3 \pm 1.4$ & 1.559 & 0.124 \\
\hline VAR & $5 \pm 1.6$ & $5.4 \pm 1.2$ & 1.1 & 0.276 \\
\hline $\mathrm{MNN}$ & $403.7 \pm 93.3$ & $378.7 \pm 63$ & 1.18 & 0.243 \\
\hline
\end{tabular}

Chi-square tests and Fisher's exact test were used for categorical variables; independent-sample t-tests were used for continuous variables. BDNF: brain-derived neurotrophic factor, BDI-II: Beck Depression Inventory-II, BMI: body mass index, HF: high frequency, HR: heart rate, LF: low frequency, LF/HF: low/high frequency ratio, MNN: mean of all normal-to-normal interbeat intervals, MRR: mean of RR intervals, RSES: Rosenberg Self-Esteem Scale, TP: total power, VAR: variance of the RR intervals, VLF: very low frequency

nia have been associated with cardiac autonomic dysregulation and reduced complexity of heart rate modulation. ${ }^{31}$ Evidence indicates that the social tasks might induce intense autonomic arousal in patients with schizophrenia, with both HF and LF decreased under social stress. ${ }^{14}$ Given the potential of compromised social functioning, such as lack of social skills and disturbances in emotional regulation, patients with schizophrenia might be refractory to available treatments. ${ }^{14}$ It has been documented that laughter has several positive physiological effects, and one of the most important effects is to help individuals cope with stress and reduce anxiety. ${ }^{32}$ The effect had been noticed to influence the hormone response in the pituitary, such as endorphins, and improved blood circulation, and decreased stress. ${ }^{33}$ In brief, we found an increased LF (a composition of both parasympathetic and sympathetic activity) in the LQP group, which is an intriguing finding and has not been observed in previous studies. This suggest that LQP may be a kind of cognitive-behavioral therapy for stress coping and bring promising the impact of rehabilitative outcomes on such patients.

Previous studies have reported that physical exercise is a specific stressor that increases secretion of cortisol, a glucocorticoid hormone secreted by the adrenal cortex, in the human body. ${ }^{34}$ The high circulating levels of cortisol signals the anterior pituitary gland to decrease adrenocorticotropic hormone (ACTH) secretion in the hypothalamic-pituitary-adrenal axis. Cortisol levels increase in proportion to the intensity of exercise, but reach an upper limit depending on the total duration of an exercise session. ${ }^{35}$ In addition, previous studies have shown that regular exercise increases basal cortisol levels and the cortisol response is likely to be dependent on the intensity and duration of the exercise, with greater intensity and longer dura- 
Table 2. Effects of 8-week simulated laughter program on physiological and psychological measures in laughter group

\begin{tabular}{|c|c|c|c|c|c|c|c|}
\hline \multirow{3}{*}{$\overline{\mathrm{BDNF}}(\mathrm{ng} / \mathrm{mL})$} & \multicolumn{2}{|c|}{ Laughter group $(\mathrm{N}=32)$} & \multicolumn{2}{|c|}{ Control group $(\mathrm{N}=27)$} & \multicolumn{3}{|c|}{ Two-way ANOVAs } \\
\hline & Week 0 & Week 8 & Week 0 & Week 8 & Treatment & Group & Treatment ${ }^{*}$ group \\
\hline & $6.3 \pm 2$ & $12.1 \pm 3.4^{\dagger}$ & $6 \pm 2.1$ & $5.5 \pm 1.7$ & $\begin{array}{l}\mathrm{F}_{1,57}=40.731 \\
\mathrm{p}<0.001\end{array}$ & $\begin{array}{l}\mathrm{F}_{1,57}=40.336 \\
\mathrm{p}<0.001\end{array}$ & $\begin{array}{l}\mathrm{F}_{1,57}=32.440 \\
\mathrm{p}<0.001\end{array}$ \\
\hline Cortisol (ng/mL) & $13 \pm 3.9$ & $13.1 \pm 3.1$ & $14.3 \pm 4$ & $14.5 \pm 4.4$ & $\begin{array}{l}\mathrm{F}_{1,57}=0.0552 \\
\mathrm{p}=0.815\end{array}$ & $\begin{array}{l}\mathrm{F}_{1,57}=2.234 \\
\mathrm{p}=0.138\end{array}$ & $\begin{array}{l}\mathrm{F}_{1,57}=0.155 \\
\mathrm{p}=0.694\end{array}$ \\
\hline BDI-II & $10.5 \pm 10.6$ & $10.2 \pm 6.5$ & $10.4 \pm 9.2$ & $9.4 \pm 6.9$ & $\begin{array}{l}\mathrm{F}_{1,57}=0.162 \\
\mathrm{p}=0.688\end{array}$ & $\begin{array}{l}\mathrm{F}_{1,57}=0.0480 \\
\mathrm{p}=0.827\end{array}$ & $\begin{array}{l}F_{1,57}=0.0268 \\
p=0.870\end{array}$ \\
\hline RSES & $22.7 \pm 1.8$ & $21.4 \pm 2.6^{*}$ & $22.9 \pm 2.9$ & $21.6 \pm 4.2$ & $\begin{array}{l}\mathrm{F}_{1,57}=4.682 \\
\mathrm{p}=0.033\end{array}$ & $\begin{array}{l}\mathrm{F}_{1,57}=0.528 \\
\mathrm{p}=0.469\end{array}$ & $\begin{array}{l}\mathrm{F}_{1,57}=0.212 \\
\mathrm{p}=0.646\end{array}$ \\
\hline Heart rate variabil & & & & & & & \\
\hline Heart rate & $90.7 \pm 12.3$ & $93.3 \pm 13.7$ & $88 \pm 16.1$ & $85.7 \pm 11.8$ & $\begin{array}{l}\mathrm{F}_{1,57}=0.0517 \\
\mathrm{p}=0.821\end{array}$ & $\begin{array}{l}\mathrm{F}_{1,57}=3.136 \\
\mathrm{p}=0.079\end{array}$ & $\begin{array}{l}\mathrm{F}_{1,57}=0.594 \\
\mathrm{p}=0.443\end{array}$ \\
\hline MRR & $712.4 \pm 139.9$ & $704 \pm 97.1$ & $738 \pm 99.8$ & $745.4 \pm 120$ & $\begin{array}{l}\mathrm{F}_{1,57}=1.759 \\
\mathrm{p}=0.187\end{array}$ & $\begin{array}{l}\mathrm{F}_{1,57}=9.041 \\
\mathrm{p}=0.003\end{array}$ & $\begin{array}{l}\mathrm{F}_{1,57}=2.048 \\
\mathrm{p}=0.155\end{array}$ \\
\hline VLF & $4.2 \pm 1.5$ & $4.4 \pm 1.1$ & $4.7 \pm 1.4$ & $4.3 \pm 2.2$ & $\begin{array}{l}\mathrm{F}_{1,57}=0.0572 \\
\mathrm{p}=0.811\end{array}$ & $\begin{array}{l}\mathrm{F}_{1,57}=0.491 \\
\mathrm{p}=0.485\end{array}$ & $\begin{array}{l}\mathrm{F}_{1,57}=0.690 \\
\mathrm{p}=0.408\end{array}$ \\
\hline LF & $2.4 \pm 2.2$ & $3 \pm 1.7^{*}$ & $3.2 \pm 1.6$ & $1.8 \pm 5.5$ & $\begin{array}{l}\mathrm{F}_{1,57}=1.592 \\
\mathrm{p}=0.210\end{array}$ & $\begin{array}{l}\mathrm{F}_{1,57}=1.508 \\
\mathrm{p}=0.222\end{array}$ & $\begin{array}{l}\mathrm{F}_{1,57}=0.280 \\
\mathrm{p}=0.598\end{array}$ \\
\hline $\mathrm{HF}$ & $2.5 \pm 1.9$ & $3 \pm 1.3$ & $3.3 \pm 1.6$ & $2.6 \pm 1.6$ & $\begin{array}{l}\mathrm{F}_{1,57}=0.616 \\
\mathrm{p}=0.434\end{array}$ & $\begin{array}{l}\mathrm{F}_{1,57}=3.415 \\
\mathrm{p}=0.067\end{array}$ & $\begin{array}{l}\mathrm{F}_{1,57}=1.320 \\
\mathrm{p}=0.253\end{array}$ \\
\hline $\mathrm{LF} / \mathrm{HF}$ & $1.8 \pm 2.6$ & $1.4 \pm 1.4$ & $1.4 \pm 1.3$ & $3.9 \pm 10.4$ & $\begin{array}{l}\mathrm{F}_{1,57}=1.903 \\
\mathrm{p}=0.170\end{array}$ & $\begin{array}{l}\mathrm{F}_{1,57}=1.363 \\
\mathrm{p}=0.245\end{array}$ & $\begin{array}{l}\mathrm{F}_{1,57}=0.790 \\
\mathrm{p}=0.376\end{array}$ \\
\hline $\mathrm{TP}$ & $4.7 \pm 1.6$ & $5 \pm 1.1$ & $5.3 \pm 1.4$ & $5 \pm 1.8$ & $\begin{array}{l}\mathrm{F}_{1,57}=0.001 \\
\mathrm{p}=0.975\end{array}$ & $\begin{array}{l}\mathrm{F}_{1,57}=1.182 \\
\mathrm{p}=0.279\end{array}$ & $\begin{array}{l}\mathrm{F}_{1,57}=1.316 \\
\mathrm{p}=0.254\end{array}$ \\
\hline VAR & $5 \pm 1.6$ & $5.3 \pm 0.9$ & $5.4 \pm 1.2$ & $5.8 \pm 1.1$ & $\begin{array}{l}\mathrm{F}_{1,57}=2.856 \\
\mathrm{p}=0.094\end{array}$ & $\begin{array}{l}\mathrm{F}_{1,57}=3.942 \\
\mathrm{p}=0.049\end{array}$ & $\begin{array}{l}\mathrm{F}_{1,57}=0.0319 \\
\mathrm{p}=0.859\end{array}$ \\
\hline MNN & $403.7 \pm 93.3$ & $405.3 \pm 71.4$ & $378.7 \pm 63$ & $335.1 \pm 108.5$ & $\begin{array}{l}\mathrm{F}_{1,57}=1.759 \\
\mathrm{p}=0.187\end{array}$ & $\begin{array}{l}\mathrm{F}_{1,57}=9.041 \\
\mathrm{p}=0.003\end{array}$ & $\begin{array}{l}\mathrm{F}_{1,57}=2.048 \\
\mathrm{p}=0.155\end{array}$ \\
\hline
\end{tabular}

Significance indicates change from pre-treatment condition in a paired t test (two-tailed). ${ }^{*} \mathrm{p}<0.05,{ }^{\dagger} \mathrm{p}<0.001$. BDNF: brain-derived neurotrophic factor, BDI-II: Beck Depression Inventory-II, BMI: body mass index, HF: high frequency, LF: low frequency, LF/HF: low/high frequency ratio, MNN: mean of all normal-to-normal interbeat intervals, MRR: mean of RR intervals, RSES: Rosenberg Self-Esteem Scale, TP: total power, VAR: variance of the RR intervals, VLF: very low frequency
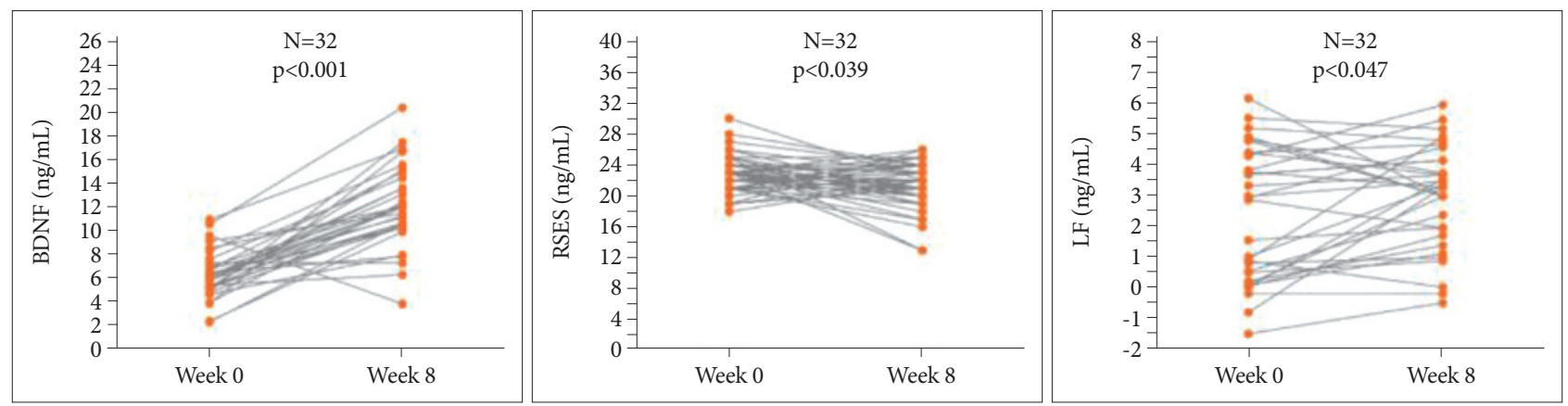

Figure 1. Significant difference of parameters examined by paired t-test in laughter group. BDNF: brain-derived neurotrophic factor, RSES: Rosenberg Self-Esteem Scale, LF: low frequency. 
tion of exercise inducing a stronger cortisol response. ${ }^{36}$ In the present study, the cortisol level at the end of the 8-week LQP was not significantly elevated in the subjects with schizophrenia. Instead, the cortisol level increased four weeks after ceasing the intervention program. One possible explanation is that restoration of a dysfunctional HPA axis might have delayed the response due to hormone production. An alternative, not mutually exclusive explanation for the delayed effect of LQP in schizophrenia is that this is a consequence of appropriate appraisal of social interaction, rather than physical effect. Albus et al. ${ }^{37}$ discovered that patients with chronic schizophrenia have a blunted or absent cortisol response not only to physical stressors but also to psychological distress. A previous study found that blunted reactions applied only specifically to psychosocial stress but not to physical stress, and the cortisol level was negatively correlated with coping strategies in patients

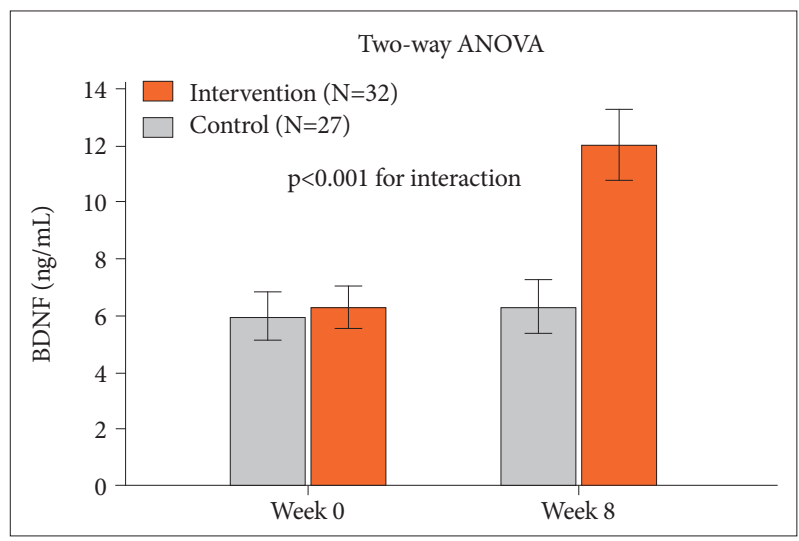

Figure 2. Level of BDNF before and after different treatment. $P$ values refer to interaction effects as measured by repeated measures analysis of variance. Error bars indicate the standard deviation. BDNF: brain-derived neurotrophic factor. with schizophrenia. ${ }^{38}$ Therefore, we suggested that LQP could improve social recognition and social interaction skills, and the secretion of cortisol might support the idea of normalization of the HPA axis in response to psychosocial stress.

It is noted that all of our subjects were prescribed psychotropic pharmacological agents such as antipsychotics, anticholinergics, benzodiazepine, lithium, valproic acid, or beta-blockers. Evidence suggests that second-generation antipsychotics, like olanzapine, quetiapine, and clozapine decrease ACTH and cortisol levels, likely through their serotonergic, adrenergic, and histaminergic pathway activities in healthy subjects. ${ }^{39}$ The diminished cortisol response could be understood as a symptomrelated effect showing desensitization of the HPA system by recurrent "environmental" stress. Moreover, after discontinuing the program, healthy people can continue the intervention program by themselves. However, the present group was administered the intervention program in a hospital. After discontinuation, the possibility of self-motivated LQP may be low based on the negative symptoms that are an essential feature of schizophrenia.

\section{Limitations}

This study has several limitations. First, all the participants were recruited from the chronic rehabilitation ward, which suggests that their psychiatric situation was controlled and relatively stable. Because we did not test the cognitive and clinical psychiatric symptoms of these patients, the generalizability to other stages of schizophrenia, such as the acute or remission states, might be limited. In addition, the negative symptoms of schizophrenia might confound the results and measures of impaired humor comprehension and humor appreciation during laughter. Second, we did not control the metabolic profile

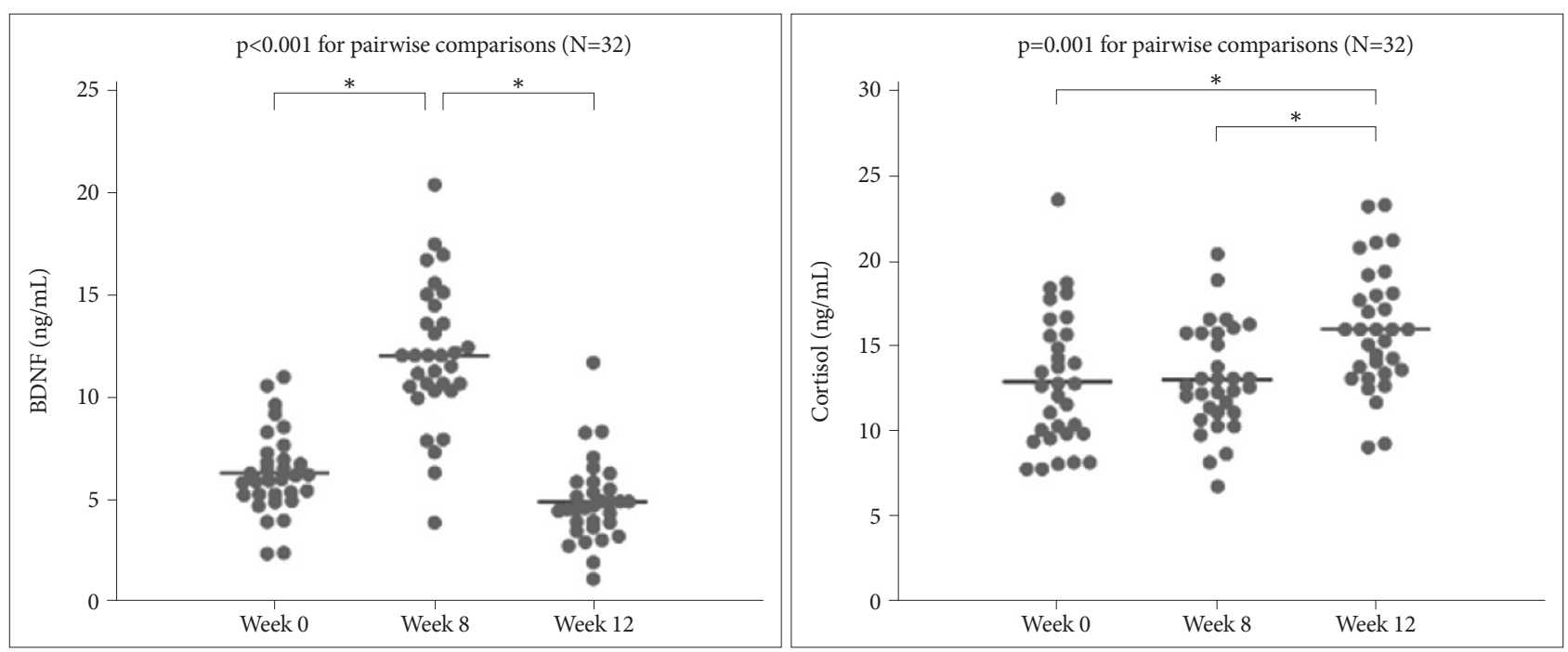

Figure 3. Incongruent expression of BDNF and cortisol after discontinuation of simulated laughter intervention. BDNF: brain-derived neurotrophic factor. ${ }^{*} p<0.05$. 
such as fasting glucose and total cholesterol of the subjects, which could have introduced bias. Third, we did not adjust for the category and doses of psychotropic agents, and the effects of various psychotropic agents on BDNF and cortisol might be different. Fourth, the intensity and duration of each LQP session may be too low in comparison to general aerobic exercise to have an influence. Fifth, our study only involved a small sample size; further investigations with larger sample sizes are needed.

In conclusion, although patients with schizophrenia experience psychotic and cognitive symptom-related social recognition deficits, LQP may increase their levels of BDNF and cortisol. LQP is an easy and available humor intervention for humor skill training that requires no additional equipment in a mental health setting. It provides a non-invasive and nonpharmacological method to treat mood symptoms, and it regulates stress level and provides social support. LQP can be considered an alternative and complementary treatment for rehabilitation of people with schizophrenia.

\section{Supplementary Materials}

The online-only Data Supplement is available with this article at https://doi.org/10.30773/pi.2020.0269.

\section{Acknowledgments}

This study was funded by the Civilian Administration Division of Beitou Branch, Tri-Service General Hospital, National Defense Medical Center (BAFH-101-02) and Penghu Branch, Tri-Service General Hospital, National Defense Medical Center (TSGH-PH-E 109011).

\section{Conflicts of Interest}

The authors have no potential conflicts of interest to disclose.

\section{Author Contributions}

Conceptualization: Ta-Chuan Yeh, Chih-Sung Liang. Data curation: Shu-Li Cheng, Ta-Chuan Yeh, Chih-Sung Liang. Formal analysis: Ta-Chuan Yeh, Chih-Sung Liang. Funding acquisition: Shu-Li Cheng. Investigation: Yu-Ting Tseng, Shih-Chieh Ku. Methodology: Fu-Chi Yang, HsuanTe Chu. Project administration: Hsuan-Te Chu, Chia-Kuang Tsai. Resources: Ta-Chuan Yeh, Chih-Sung Liang. Software: Chia-Kuang Tsai. Supervision: Chih-Sung Liang. Validation: Shu-Li Cheng, Fu-Chi Yang, Ta-Chuan Yeh, Chih-Sung Liang. Visualization: Shih-Chieh Ku, Yu-Ting Tseng. Writingoriginal draft: Shu-Li Cheng.Writing-review \& editing: Ta-Chuan Yeh, Chih-Sung Liang.

\section{ORCID iDs}

$\begin{array}{ll}\text { Shu-Li Cheng } & \text { https://orcid.org/0000-0002-1523-8519 } \\ \text { Fu-Chi Yang } & \text { https://orcid.org/0000-0001-6831-3634 } \\ \text { Hsuan-Te Chu } & \text { https://orcid.org/0000-0003-3722-3286 } \\ \text { Chia-Kuang Tsai } & \text { https://orcid.org/0000-0001-7693-1408 } \\ \text { Shih-Chieh Ku } & \text { https://orcid.org/0000-0002-2044-3402 } \\ \text { Yu-Ting Tseng } & \text { https://orcid.org/0000-0002-9338-8344 } \\ \text { Ta-Chuan Yeh } & \text { https://orcid.org/0000-0002-1016-3634 } \\ \text { Chih-Sung Liang } & \text { https://orcid.org/0000-0003-1138-5586 }\end{array}$

\section{REFERENCES}

1. Bryant GA, Aktipis CA. The animal nature of spontaneous human laughter. Evol Hum Behav 2014;35:327-335.

2. Mora-Ripoll R. Potential health benefits of simulated laughter: a narrative review of the literature and recommendations for future research. Complement Ther Med 2011;19:170-177.

3. Mora-Ripoll R. The therapeutic value of laughter in medicine. Altern Ther Health Med 2010;16

4. Kim SH, Kim YH, Kim HJ. Laughter and stress relief in cancer patients: a pilot study. Evid Based Complement Alternat Med 2015;2015: 864739 .

5. Manninen S, Tuominen L, Dunbar RI, Karjalainen T, Hirvonen J, Arponen E, et al. Social laughter triggers endogenous opioid release in humans. J Neurosci 2017;37:6125-6131.

6. Kahn RS, Sommer IE, Murray RM, Meyer-Lindenberg A, Weinberger DR, Cannon TD, et al. Schizophrenia. Nat Rev Dis Primers 2015;1: 15067.

7. Murri MB, Pariante CM, Dazzan P, Hepgul N, Papadopoulos AS, Zunszain $\mathrm{P}$, et al. Hypothalamic-pituitary-adrenal axis and clinical symptoms in first-episode psychosis. Psychoneuroendocrinology 2012;37: 629-644.

8. Aas M, Dazzan P, Mondelli V, Toulopoulou T, Reichenberg A, Di Forti $\mathrm{M}$, et al. Abnormal cortisol awakening response predicts worse cognitive function in patients with first-episode psychosis. Psychol Med 2011;41:463-476.

9. Mondelli V, Dazzan P, Hepgul N, Di Forti M, Aas M, D’Albenzio A, et al. Abnormal cortisol levels during the day and cortisol awakening response in first-episode psychosis: the role of stress and of antipsychotic treatment. Schizophr Res 2010;116:234-242.

10. Fujibayashi M, Matsumoto T, Kishida I, Kimura T, Ishii C, Ishii N, et al. Autonomic nervous system activity and psychiatric severity in schizophrenia. Psychiatry Clin Neurosci 2009;63:538-545.

11. Iwamoto Y, Kawanishi C, Kishida I, Furuno T, Fujibayashi M, Ishii C, et al. Dose-dependent effect of antipsychotic drugs on autonomic nervous system activity in schizophrenia. BMC Psychiatry 2012;12:199.

12. Kim JH, Yi SH, Yoo CS, Yang SA, Yoon SC, Lee KY, et al. Heart rate dynamics and their relationship to psychotic symptom severity in clozapine-treated schizophrenic subjects. Prog Neuropsychopharmacol Biol Psychiatry 2004;28:371-378.

13. Bär KJ, Rachow T, Schulz S, Bassarab K, Haufe S, Berger S, et al. The phrenic component of acute schizophrenia--a name and its physiological reality. PLoS One 2012;7:e33459.

14. Jáuregui OI, Costanzo EY, de Achával D, Villarreal MF, Chu E, Mora $\mathrm{MC}$, et al. Autonomic nervous system activation during social cognition tasks in patients with schizophrenia and their unaffected relatives. Cogn Behav Neurol 2011;24:194-203.

15. Green M, Matheson S, Shepherd A, Weickert C, Carr V. Brain-derived neurotrophic factor levels in schizophrenia: a systematic review with meta-analysis. Mol Psychiatry 2011;16:960-972.

16. Fernandes B, Steiner J, Berk M, Molendijk M, Gonzalez-Pinto A, Turck C, et al. Peripheral brain-derived neurotrophic factor in schizophrenia and the role of antipsychotics: meta-analysis and implications. Mol Psychiatry 2015;20:1108-1119.

17. Huang EJ, Reichardt LF. Neurotrophins: roles in neuronal development and function. Annu Rev Neurosci 2001;24:677-736.

18. van Haren NE, Cahn W, Pol HH, Kahn R. Schizophrenia as a progressive brain disease. Eur Psychiatry 2008;23:245-254.

19. Niitsu T, Shirayama Y, Matsuzawa D, Hasegawa T, Kanahara N, Hashimoto T, et al. Associations of serum brain-derived neurotrophic factor with cognitive impairments and negative symptoms in schizophrenia. Prog Neuropsychopharmacol Biol Psychiatry 2011;35:1836-1840.

20. Favalli G, Li J, Belmonte-de-Abreu P, Wong AH, Daskalakis ZJ. The role of BDNF in the pathophysiology and treatment of schizophrenia. J Psychiatr Res 2012;46:1-11.

21. Cai C, Yu L, Rong L, Zhong H. Effectiveness of humor intervention for patients with schizophrenia: a randomized controlled trial. J Psychiatr Res 2014;59:174-178. 
22. Knapp M, Mangalore R, Simon J. The global costs of schizophrenia. Schizophr Bull 2004; 30: 279-293.

23. Chang C, Tsai G, Hsieh CJ. Psychological, immunological and physiological effects of a Laughing Qigong Program (LQP) on adolescents. Complement Ther Med 2013;21:660-668.

24. Hsieh CJ, Chang C, Tsai G, Wu HF. Empirical study of the influence of a Laughing Qigong Program on long-term care residents. Geriatr Gerontol Int 2015;15:165-173.

25. Kuo TB, Lin T, Yang CC, Li CL, Chen CF, Chou P. Effect of aging on gender differences in neural control of heart rate. Am J Physiol 1999; 277:H2233-2239.

26. Hsu CC, Liang CS, Tai YM, Cheng SL. Incongruent changes in heart rate variability and body weight after discontinuing aerobic exercise in patients with schizophrenia. Int J Psychophysiol 2016;109:132-137.

27. Rosenberg M. Society and the Adolescent Self-Image, Revised Edition. Middletown, CT: Wesleyan University Press; 1989.

28. Beck AT, Ward CH, Mendelson M, Mock J, Erbaugh J. An inventory for measuring depression. Arch Gen Psychiatry 1961;4:561-571.

29. Kim HJ, Song BK, So B, Lee O, Song W, Kim Y. Increase of circulating BDNF levels and its relation to improvement of physical fitness following 12 weeks of combined exercise in chronic patients with schizophrenia: a pilot study. Psychiatry Res 2014;220:792-796.

30. Szuhany KL, Bugatti M, Otto MW. A meta-analytic review of the effects of exercise on brain-derived neurotrophic factor. J Psychiatr Res 2015;60:56-64.
31. Montaquila JM, Trachik BJ, Bedwell JS. Heart rate variability and vagal tone in schizophrenia: a review. J Psychiatr Res 2015;69:57-66.

32. Cha MY, Hong HS. Effect and path analysis of laughter therapy on serotonin, depression and quality of life in middle-aged women. J Korean Acad Nurs 2015;45:221-230.

33. Yim J. Therapeutic benefits of laughter in mental health: a theoretical review. Tohoku J Exp Med 2016;239:243-249.

34. Hackney AC. Stress and the neuroendocrine system: the role of exercise as a stressor and modifier of stress. Expert Rev Endocrinol Metab 2006;1:783-792.

35. Davies C, Few J. Effects of exercise on adrenocortical function. J Appl Physiol 1973;35:887-891.

36. Hill E, Zack E, Battaglini C, Viru M, Viru A, Hackney A. Exercise and circulating cortisol levels: the intensity threshold effect. J Endocrinol Invest 2008;31:587-591.

37. Albus M, Ackenheil M, Engel RR, Müller F. Situational reactivity of autonomic functions in schizophrenic patients. Psychiatry Res 1982;6: 361-370.

38. Jansen LM, Gispen-de Wied CC, Kahn RS. Selective impairments in the stress response in schizophrenic patients. Psychopharmacology 2000;149:319-325.

39. Cohrs S, Röher C, Jordan W, Meier A, Huether G, Wuttke W, et al. The atypical antipsychotics olanzapine and quetiapine, but not haloperidol, reduce ACTH and cortisol secretion in healthy subjects. Psychopharmacology 2006; 185:11-18. 


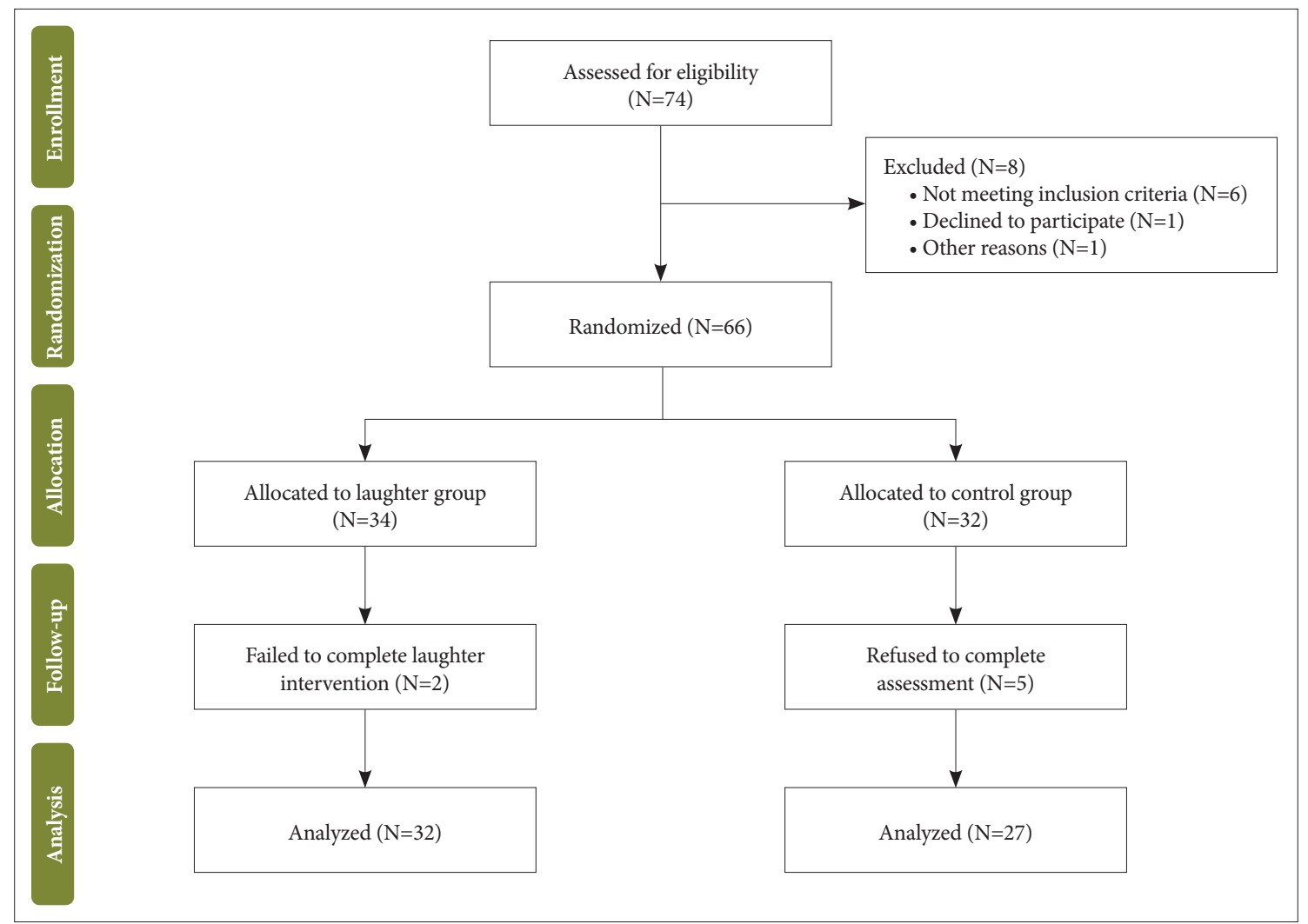

Supplementary Figure 1. CONSORT diagram of the trial. 